\title{
A STATISTICS-BASED MATERIAL PROPERTY ANALYSIS TO SUPPORT ABLATION SIMULATION UQ EFFORTS
}

\author{
Sean R. Copeland and Juan Alonso \\ Department of Aeronautics \& Astronautics \\ Stanford University, Stanford, CA \\ sean. copelandestanford.edu
}

\author{
Milad Mahzari \\ Guggenheim School of Aerospace Engineering \\ Georgia Institute of Technology, Atlanta, GA \\ mmahzari3egatech.edu
}

\section{Ioana Cozmuta}

\author{
Advanced Supercomputing Division \\ STC/NASA Ames, Moffett Field, CA \\ Ioana. Cozmuta@nasa.gov
}

\begin{abstract}
Accurate characterization of entry capsule heat shield material properties is a critical component in modeling and simulating Thermal Protection System (TPS) response in a prescribed aerothermal environment. The thermal decomposition of the TPS material during the pyrolysis and charring processes is typically poorly characterized and results in large uncertainties in material properties as inputs for ablation models. These material property uncertainties contribute to large design margins on flight systems and cloud re-construction efforts for data collected during flight and ground testing, making revision to existing models for entry systems more challenging. This work focuses on the following areas of interest to the ablation modeling community: a proper characterization of input probability density functions for material properties, an uncertainty propagation to identify how the uncertainties affect quantities of interest, a sensitivity and uncertainty contributor breakdown, and an analysis of how errors in input characterization contribute to errors in output distributions.
\end{abstract}

\section{INTRODUCTION}

For the past half-century there has existed a need to accurately predict in-depth temperatures and surface recession levels for ablative TPS materials to perform design and analysis of entry systems. These predictions are subject to various errors and uncertainties that can be classified into the following categories:

1. Aleatory - Uncertainties due to natural variations in the TPS material properties or aerothermal environmental conditions.

2. Epistemic - Errors arising from improper input characterization and/or an inability to properly capture the physics of the problem with the ablation model governing equations or numerical methods.

Aleatory and epistemic uncertainties corrupt the accuracy of the predicted outputs and can lead to erroneous conclusions. Expert judgement, experience and good engineering practice mitigate the potential consequences of these uncertainties, but this approach falls short when the system design, aerothermal environment and entry trajectory deviates from established practice.

Over the past decade, numerous analyses have been performed using a probabilistic interpretation of aleatory uncertainty. Monte Carlo methodologies have been applied to uncertain parameters in ablation[9, 6, 17] and aerothermal[2, 3] models, to determine the effect on imposed heating, subsurface temperatures, surface recession levels and other quantities of interest. These approaches have given rise to new methods for assessing TPS margin[8] and have been the basis for new design and risk assessment methodologies. However, as is the case in deterministic simulations, the outputs from stochastic analyses depend strongly on the characterization of the input distribution(s). In the ablation modeling community, little work has been performed to collect and analyze the available material property data to arrive at appropriate input distributions that capture the correct trends and relationships between material properties. In the works cited, all Monte Carlo analyses are performed 
assuming independent gaussian random variables as inputs with distribution parameters $(\mu$ and $\sigma)$ determined by expert opinion. This particular prescription of input functional type can lead to improper and non-physical inputs to the ablation models. Furthermore, there has been little quantitative analysis to determine how the assumed input PDF shapes affect the shape of the output PDF and any corresponding conclusions on reliability and margin.

This work addresses some of the highlighted shortcomings in the existing body of work for uncertainty quantification efforts for ablation simulations. Primary goals are outlined below:

1. Construct a revised set of input material property PDFs for modern TPS materials. Known dependencies exist between material properties (density, thermal conductivity, specific heat, etc.) and should be appropriately treated in nondeterministic analyses. A prescription of the functional type of the input PDF is to be avoided and instead will be based on a Kriging fit to available experimental data. Results from NASA's Mars Science Laboratory (MSL) TPS design and MSL Entry, Descent and Landing Instrumentation (MEDLI) material property testing efforts form the database for the analysis.

2. Propagate input uncertainties to output quantities of interest, perform model sensitivity analysis and rank the primary uncertainty contributors. The propagation analysis determines the effect of the revised input set on typical quantities of interest, including in-depth temperature, bond line temperature, and recession depth. The model sensitivity study is performed using standard finite-difference methods while uncertainty contributors are determined by calculating correlation coefficients and Sobol indices.

3. Characterize the errors output PDFs as functions of the quality of the input distributions. A quantitative assessment of the affect of additional material property data on output error reduction is performed. A specified number of samples taken from the input PDFs generated in (1) (assumed to be "truth" distributions) are used to create approximate input distributions to be used in a Monte Carlo ablation analysis. Output PDFs from the approximate models are compared to "true" output PDFs on the basis of Kullback-Liebler divergence. This framework establishes a mapping between the number of samples used to generate the approximate inputs and the error in the predicted outputs.

The ablation analysis is performed using the Fully Implicit Ablation and Thermal Response Program (FIAT)[5] developed and used by NASA to perform analysis and design for flight systems.

\section{PRELIMINARY RESULTS}

The ablation simulations in this abstract are calculated in aerothermal environments anticipated for the Mars Science Laboratory during the entry phase of its mission, scheduled for the summer of 2012. The sensitivity study was performed at two locations on the heat shield corresponding to embedded instrumentation, called MEDLI Integrated Sensor Plugs (MISPs). Each plug has four in-depth thermocouples (TCs) that will track the evolution of temperature through the TPS during entry.

The finite-difference sensitivity analysis for MISP locations $3 \& 4$ at each thermocouple locations are shown in Fig. (1) and Fig. (2) respectively. The stacked-area plots show the relative sensitivity of the TC temperature to the 11 material property inputs to FIAT. For a given vertical slice along the x-axis, the fraction of color belonging to parameters indicate how significant those parameters are at that time step. For an example, see Fig. (1a). The sensitivity of TC1 temperature at MISP 3 to char density is zero until the onset of char formation 33 seconds past entry interface where it then contributes significantly to the sensitivity of the TC reading. Note the time axis on Fig. (1a) differs from the other subfigures due to the burnout of the thermocouple as the TPS material recedes beyond its in-depth location in the plug.

In anticipation of true TPS material property data, preliminary uncertainty propagation has been performed using fictitious input distributions. This propagation has been performed with independent gaussian and uniform random variables as a placeholder for more accurate input distributions that will be characterized for the final work. Propagation analyses of 2000 FIAT runs for each distribution type each are shown in Fig. (3) at MISP 3. TC mean values for each time step are shown in solid lines and a band of two standard deviations is enclosed in the dotted lines.

\section{REFERENCES}

[1] Anderson, J. D., Hypersonic and High-Temperature Gas Dynamics, AIAA Education Series, Reston, 2006.

[2] Bose, D., Wright, M., Gökçen, T., "Uncertainty and Sensitivity Analysis of Thermochemical Modeling for Titan Atmospheric Entry," AIAA 2004-2455, 37 th AIAA Thermophysics Conference, Portland, OR, 2004. 


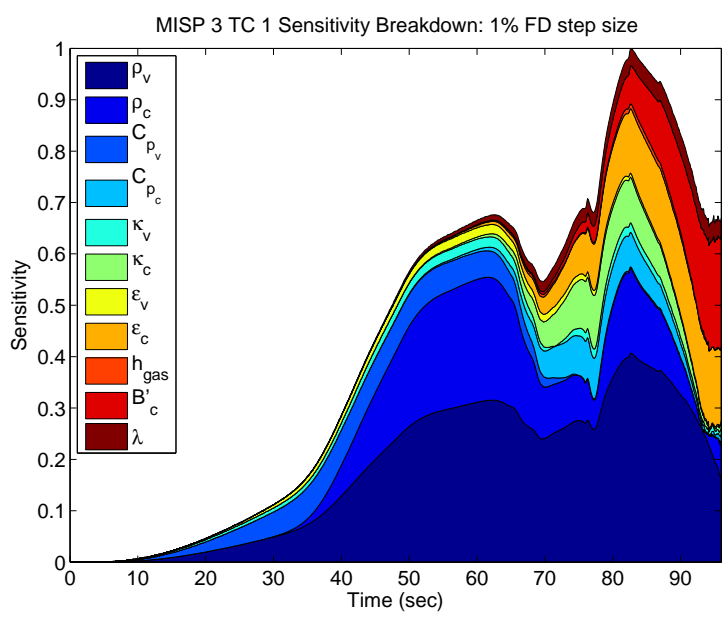

(a)

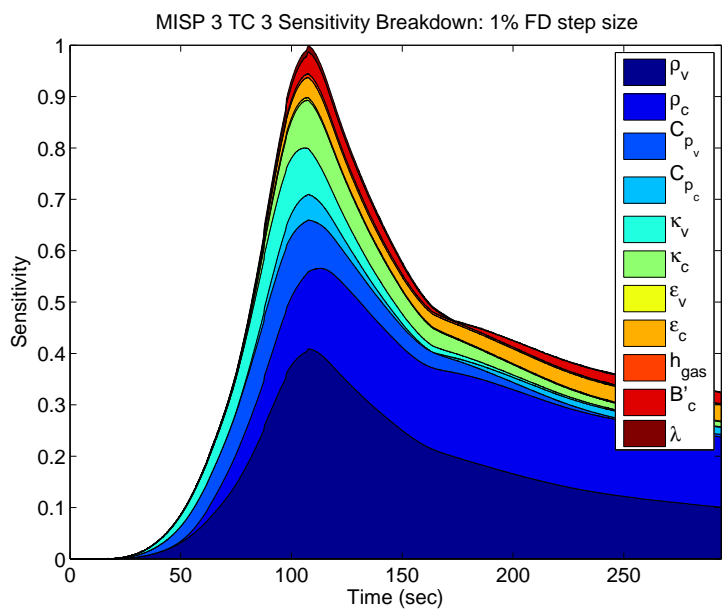

(c)

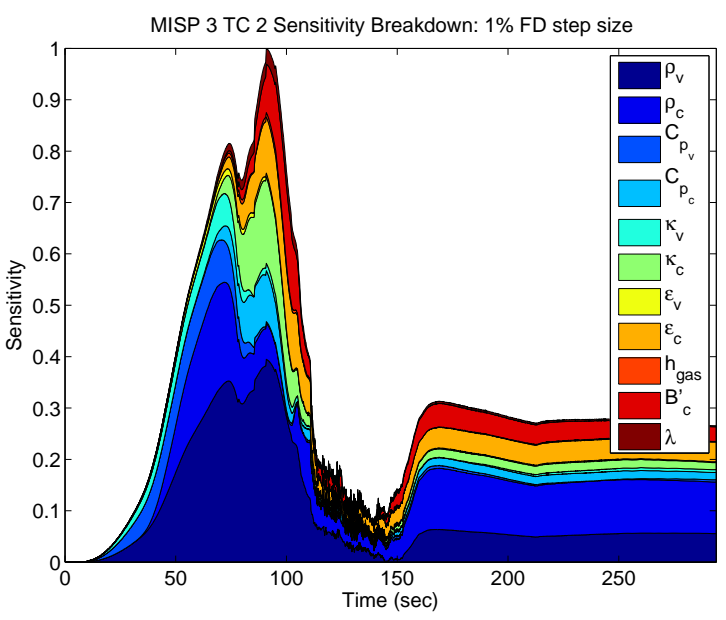

(b)

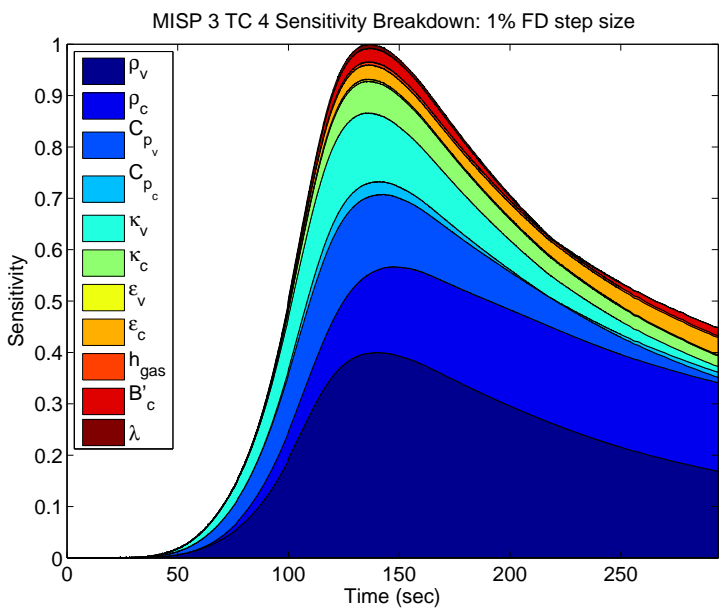

(d)

Figure 1: MISP 3 Finite-Difference Sensitivity Analysis.

[3] Bose, D., Wright, M., "Uncertainty Analysis of Laminar Aeroheating Predictions for Mars Entries," AIAA 2005-4682, $38^{\text {th }}$ AIAA Thermophysics Conference, Toronto, Ontario Canada, 2005.

[4] Chen, Y.-K., and Milos, F. S., Fully Implicit Ablation and Thermal Analysis Program (FIAT), ICCE/4, Fourth International Conference on Composites Engineering, edited by D. Hui, International Community for Composites Engineering and College of Engineering, Univ. of New Orleans, New Orleans, LA, 1997, pp. 661, 662.

[5] Chen, Y.-K., Milos, F.S., Ablation and Thermal Response Program for Spacecraft Heatshield Analysis, Journal of Spacecraft and Rockets, Vol. 36, No. 3, May-June 1999.

[6] Chen, Y.-K., Squire, T., Laub, B., Wright, M., "Monte Carlo Analysis for Spacecraft Thermal Protection System Design," AIAA 2006-2951, $9^{\text {th }}$ AIAA/ASME Joint Thermophysics and Heat Transfer Conference, San Francisco, CA, 2006.

[7] Users Manual: Aerotherm Charring Material Thermal Response and Ablation Program, Aerotherm Div., Acurex Corp., Mountain View, CA, Aug. 1987.

[8] Cozmuta, I., et. al, "Defining Ablative Thermal Protection System Margins for Planetary Entry Vehicles", AIAA 2011 3757, 42nd AIAA Thermophysics Conference, Honolulu, HI 2011. 


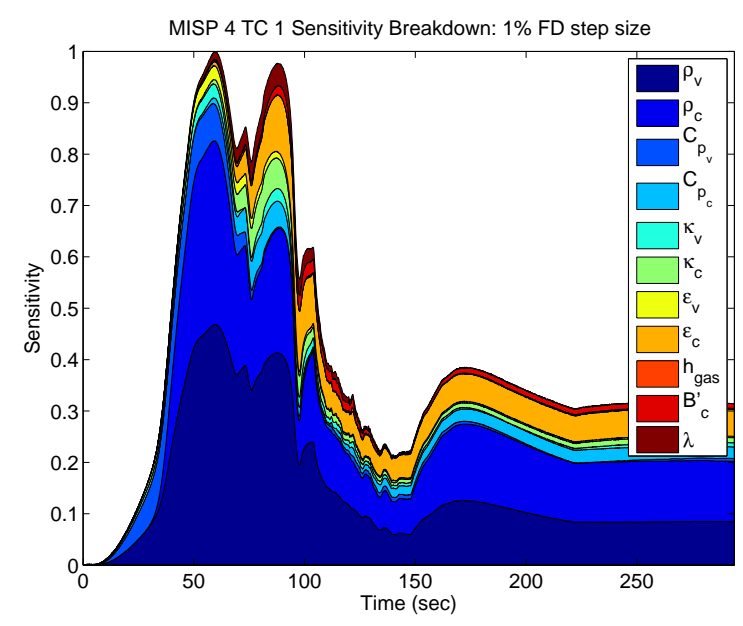

(a)

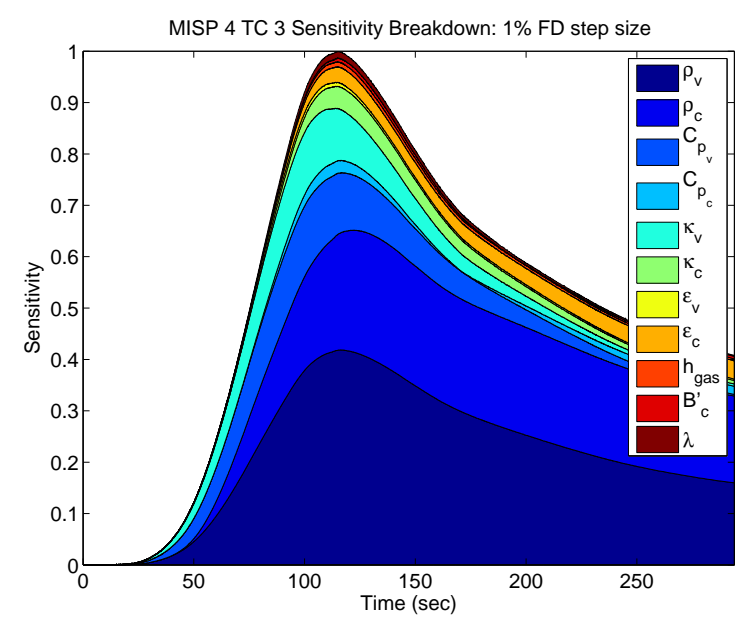

(c)

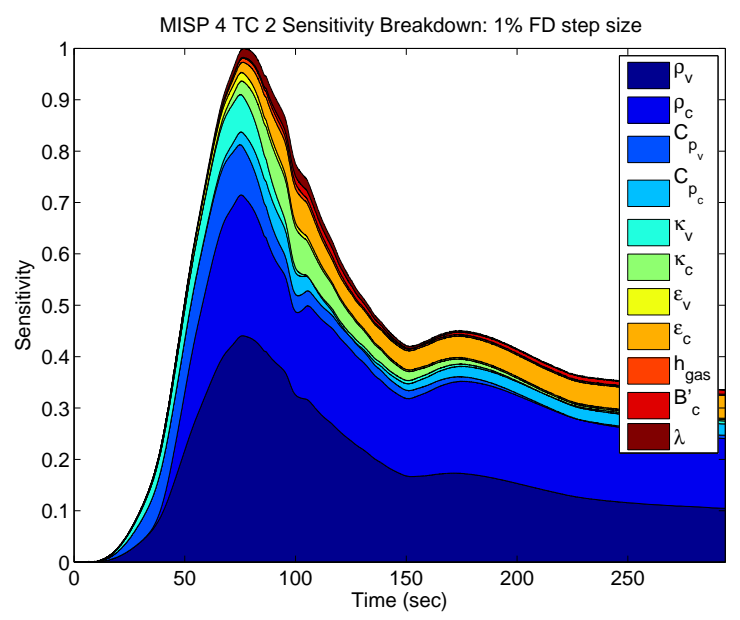

(b)

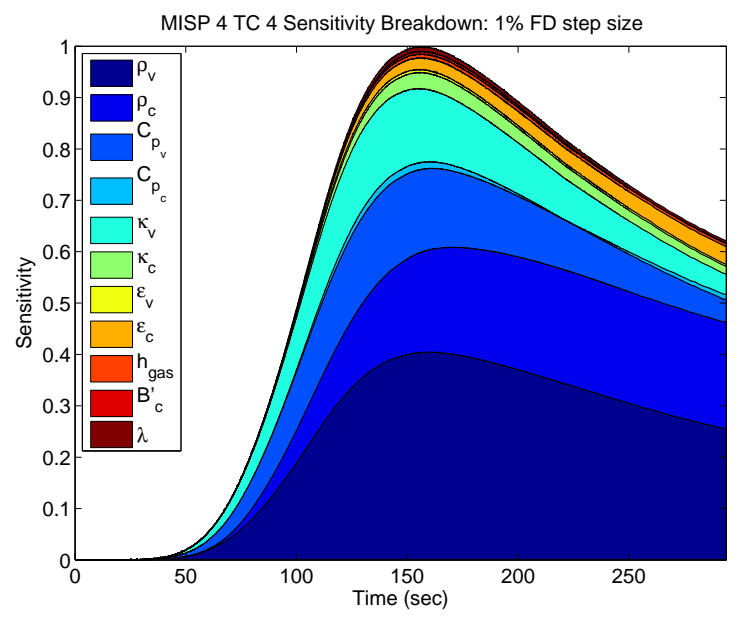

(d)

Figure 2: MISP 4 Finite-Difference Sensitivity Analysis.

[9] Dec, J. A., Mitcheltree, R. A., "Probabilistic Design of a Mars Sample Return Earth Entry Vehicle Thermal Protection System," AIAA 2002-0910, 40 ${ }^{\text {th }}$ AIAA Aerospace Sciences Meeting \& Exhibit, Reno, NV 2002.

[10] Edquist, K. T., Dyakonov, A. A., Wright, M. J., Tang, C. Y., "Aerothermodynamic Design of the Mars Science Laboratory Heatshield," AIAA 2009-4075, $41^{\text {st }}$ AIAA Thermophysics Conference, San Antonio, TX, 2009.

[11] Gazarik, M., Wright, M., Little, A., Cheatwood, F., Herath, J., Munk, M., Novak, J., and Martinez, E., Overview of the MEDLI Project, IEEE Aerospace Conference, Big Sky, MT, No. IEEEAC paper No. 1510, 2008.

[12] Laub, B., Chen, Y.-K., Dec, J. A., "Development of a High-Fidelity Thermal/Ablation Response Model for SLA-561V," AIAA 2009-4232, $41^{\text {st }}$ AIAA Thermophysics Conference, San Antonio, TX, 2009.

[13] Mansour, N., Pittman, J., Olson, L., "Fundamental Aeronautics Hypersonics Project: Overview," AIAA 2007-4263, 39 AIAA Thermophysics Conference, Miami, FL, June, 2007.

[14] McGuire, M. K., Bowles, J. V., Yang L. H., Kinney, D. J., Roberts, C. D., "TPS Selection and Sizing Tool Implemented in an Advanced Engineering Environment," AIAA 2004-342, $42^{\text {nd }}$ AIAA Aerospace Sciences Meeting \& Exhibit, Reno, NV 2004. 


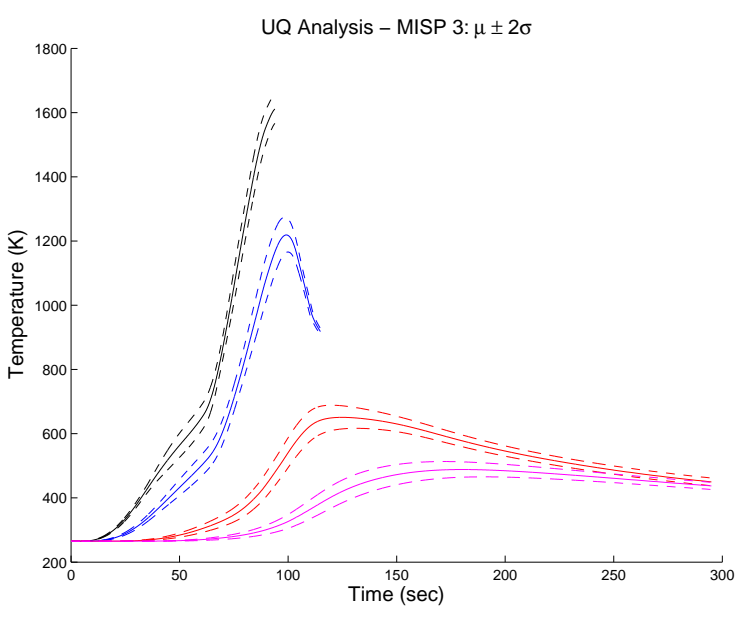

(a) Normal Distributions

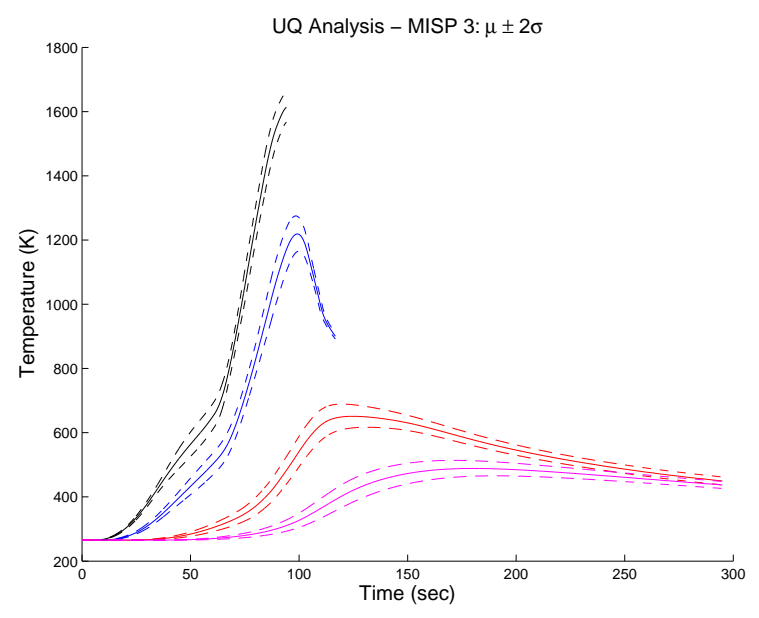

(b) Uniform Distributions

Figure 3: MISP 3 Uncertainty Quantification Analysis Using Differing Input Distributions.

[15] Moyer, C. B., and Rindal, R. A., An Analysis of the Coupled Chemically Reacting Boundary Layer and Charring Ablator, Part II, Finite Difference Solution for the In-Depth Response of Charring Materials Considering Surface Chemical and Energy Balances, NASA CR-1061, June 1968.

[16] Rasmussen, C. E., and Williams C., Gaussian Processes for Machine Learning, MIT Press, Cambridge, 2006.

[17] Sepka, S. A., Wright, M., "Monte Carlo Approach to FIAT Uncertainties with Applications for Mars Science Laboratory," Journal of Thermophysics and Heat Transfer, Vol. 25, No. 4, October-December 2011.

[18] Stackpoole, M., Sepka, S., Cozmuta, I., Kontinos, D., "Post-Flight Evaluation of Stardust Sample Return Capsule Forebody Heatshield Material," AIAA 2008-1202, 46 th AIAA Aerospace Sciences Meeting \& Exhibit, Reno, NV, 2008.

[19] White, T., et. al, "Proposed Analysis Process for Mars Science Laboratory Heat Shield Sensor Plug Flight Data", AIAA 2011-3957, 42nd AIAA Thermophysics Conference, Honolulu, HI 2011.

[20] Wright, M. J., et. al, "A Review of Aerothermal Modeling for Mars Entry Missions", AIAA 2010-443, 48th AIAA Aerospace Sciences Meeting Including the New Horizons Forum and Aerospace Exposition, Orlando, FL 2010. 